Article

\title{
Individual Identification of Cheetah (Acinonyx jubatus) Based on Close-Range Remote Sensing: First Steps of a New Monitoring Technique
}

\author{
Guillaume Baralle 1(D), Antoine F. J. Marchal ${ }^{2, *}$, Philippe Lejeune 1,2(D) and Adrien Michez ${ }^{1,3}$ \\ 1 Gembloux Agro-Bio Tech, TERRA Teaching and Research Centre (Forest is Life), University of Liège, \\ 2 Passage des Déportés, 5030 Gembloux, Belgium; guillaume.baralle@alumni.uliege.be (G.B.); \\ p.lejeune@ulg.ac.be (P.L.); adrien.michez@univ-rennes2.fr (A.M.) \\ 2 Wildlife 3D Tracking (W3DT), rue des Ponts 98, 1480 Tubize, Belgium \\ 3 University Rennes 2 LETG (CNRS UMR 6554), Place du Recteur Henri Le Moal, CEDEX, 35043 Rennes, France \\ * Correspondence: info@wildlife3dtracking.org
}

Citation: Baralle, G.; Marchal, A.F.J.; Lejeune, P.; Michez, A. Individual Identification of Cheetah (Acinonyx jubatus) Based on Close-Range Remote Sensing: First Steps of a New Monitoring Technique. Remote Sens. 2021, 13, 1090. https:// doi.org/10.3390/rs13061090

Academic Editor: Qinghua Guo

Received: 29 January 2021

Accepted: 11 March 2021

Published: 12 March 2021

Publisher's Note: MDPI stays neutral with regard to jurisdictional claims in published maps and institutional affiliations.

Copyright: (C) 2021 by the authors. Licensee MDPI, Basel, Switzerland. This article is an open access article distributed under the terms and conditions of the Creative Commons Attribution (CC BY) license (https:// creativecommons.org/licenses/by/ $4.0 /)$.

\begin{abstract}
Wildlife monitoring is an important part of the conservation strategies for certain endangered species. Non-invasive methods are of significant interest because they preserve the studied animal. The study of signs, especially tracks, seems to be a valuable compromise between reliability, simplicity and feasibility. The main objective of this study is to develop and test an algorithm that can identify individual cheetahs based on 3D track modelling using proximal sensing with an off-theshelf camera. More specifically, we propose a methodological approach allowing the identification of individuals, their sex and their foot position (i.e., left/right and hind/front). In addition, we aim to compare different track recording media: 2D photo and 3D photo models. We sampled 669 tracks from eight semi-captive cheetahs, corresponding to about 20 tracks per foot. We manually placed on each track 25 landmarks: fixed points representing the geometry of an object. We also automatically placed 130 semi-landmarks, landmark allowed to move on the surface, per track on only the 3D models. Geometric morphometrics allowed the measurement of shape variation between tracks, while linear discriminant analysis (LDA) with jack-knife prediction enabled track discrimination using the information from their size and shape. We tested a total of 82 combinations of features in terms of recording medium, landmarks configuration, extracted information and template used. For foot position identification, the best combination correctly identified $98.2 \%$ of the tracks. Regarding those results, we also ran an identification algorithm on a dataset containing only one kind of foot position to highlight the differences and mimic an algorithm identifying the foot position first and then an individual factor (here, sex and identity). This led to accuracy of 94.8 and $93.7 \%$, respectively, for sex and individual identification. These tools appear to be effective in discriminating foot position, sex and individual identity from tracks. Future works should focus on automating track segmentation and landmark positioning for ease of use in conservation strategies.
\end{abstract}

Keywords: Acinonyx jubatus; cheetah; non-invasive monitoring; close-range remote sensing; photogrammetry; track; wildlife monitoring

\section{Introduction}

Since the 1950s, the intensive use of ecosystems by humans has led to major changes in the biosphere [1]. Most of the world's biological diversity cannot adapt to such sudden transformations. In addition, mammal populations tend to decline drastically, particularly carnivores [2-4]. Our study case, the cheetah (Acinonyx jubatus), is classified as vulnerable with a total estimated population of 6674 mature individuals according to the International Union for Conservation of Nature's Red List assessment [5]. This number keeps declining [6]. Nowadays, the majority of wild cheetahs live in southern and eastern Africa, mainly outside protected areas [5]. Their current range is estimated at $10 \%$ of the 
historical range [7]. The need for data to understand and monitor cheetah populations is therefore crucial.

Wildlife monitoring enables a better understanding of animal populations. However, it often requires direct contact with the studied animal. From the most intimate contact, such as fitting GPS collars, to the most distant, such as camera trapping, monitoring can affect the animals, potentially modifying their fitness [8,9]. The possibility of indirectly monitoring wild animals without disturbing them is of significant interest. Accomplishing this is to record indirect signs of presence. These signs include feces, territorial marks and tracks [10]. Tracks are particularly interesting, because they have specific and differentiable characteristics that are related to the species, sex, age and even individual. This is highlighted by the ability of some traditional trackers to identify the species, age and sex of animals and/or individual wild animals through their tracks with a high degree of certainty $[11,12]$. For several years, study programs have been trying to use presence signs to monitor certain animal species. For tracks, successful attempts have been made, first using direct measurement [13] and, more recently using digitized measurements, in particular the FIT (Footprint Identification Technique) program [13-15]. Based on photographs of tracks, researchers were able to create individual identification algorithms for a wide range of mammal species [13-15], with some emphasis on cheetah [16].

The use of a three-dimensional (3D) recording technique is a recent advance in the field, which has been used on lions (Panthera leo) [17-19]. This new method allows the extraction of more information from the track (including depth), to decrease the manipulator bias and allow greater automation of both the recording and feature extraction processes. In order to extract information on the geometry of tracks, the 3D method uses geometric morphometrics analysis. Morphometrics is used to study the geometry of living organisms, their variations and covariations (Cucchi et al., 2015).

These techniques are based on the placement of landmarks, which are discrete points corresponding to anatomical loci that are homologous among the studied entities [20]. As landmarks sometimes do not allow the characterization of all geometric information, particularly variations in curves and surfaces, morphometricians developed semilandmarks [21]. These are discrete points that do not correspond to anatomical loci and are therefore able to slide along curves and surfaces [22], hence their designation as curve or surface-sliders. The positions of the landmarks and semilandmarks are then subjected to a generalized procrustes analysis (GPA). This superimposition projects all the landmark coordinates into a non-linear space. It uses a least-squares superposition method to minimize the distance between all homologous landmarks in the sample and thus, to isolate the shape of the studied objects [23]. The new coordinates are known as Procrustes coordinates [21]. This process eliminates the effects of size, orientation and position using an iterative algorithm in order to keep only the true differences between tracks shapes [21]. GPA creates a consensus object based on the mean position of the superimposed landmarks made out of the entire dataset. Shape variations between the objects are studied in relation to this consensus. In addition to shape, size is also analyzed by calculating the centroid size of each track. It is mathematically traduced by the square root of the sum of the squared distances to the centroid of the object and corresponds to all the information available on the size [20]. The information added from the shape and the size refers to the form [24]. The method of Marchal et al. [17,18] uses morphometrics to extract geometric information from tracks that can be used for individual identification. However, this method remains under development. Even if it successfully distinguished foot position, age and sex of lions, individual identification remains inaccurate [18].

The objective of this study is to compare different combinations of features, such as recording medium, landmark configuration, type of extracted information and template, to implement an identification algorithm for cheetah tracks. The aim is to find the most suitable set of features to identify the foot position, sex and individual with the highest accuracy. In the end, this could become a reliable, user-friendly and cost-effective tool to 
identify and sex animals in a non-invasive way that would improve wildlife monitoring, and therefore conservation strategies.

\section{Materials and Methods}

\subsection{Ethics Statement}

The cheetahs used for this experiment are legally held in captivity and registered by N/a'ankusê Wildlife Sanctuary (Khomas Region, Namibia). The animals were not restrained or harmed in any ways; their participation consisted of walking through a prepared sand patch in exchange for a food reward (Figure 1A).

A. Track sampling

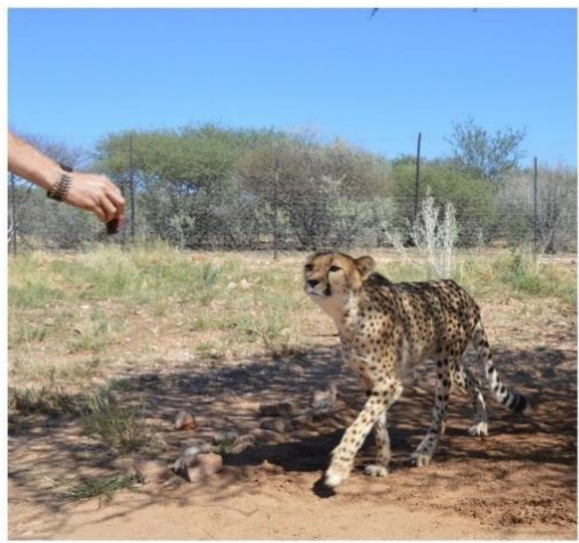

B. Image capture
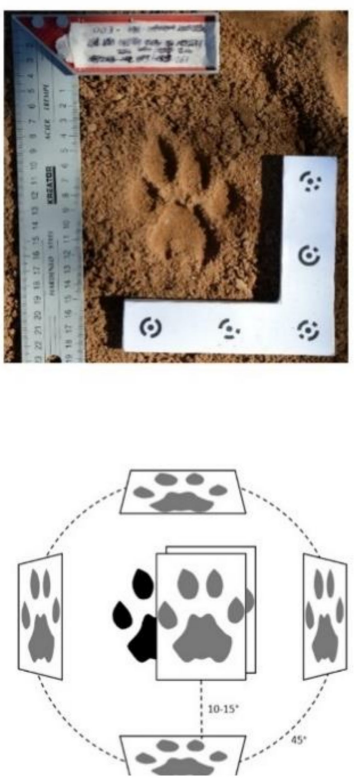

C. Landmarking

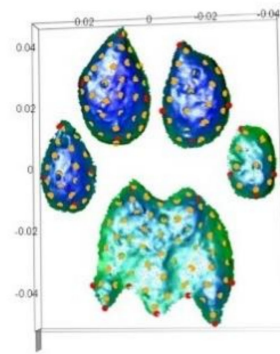

3D

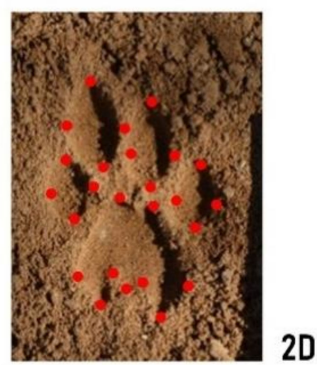

Figure 1. Track sampling and processing scheme. (A) Track sampling: the cheetah is led into sand patch in exchange for food reward. (B) Photographic track sampling protocol scheme: track is framed by rectangular centimetric ruler (2D) and 3D targets for automatic recognition in Agisoft. Rectangles represent 6 photos of sampled track (in black) needed to reconstruct 3D model by photogrammetry ( 5 pictures, from above and around track) and for 2D method ( 1 picture, from above). (C) Landmarking: 150 surface-sliders (3D) and 25 landmarks on photo (2D approach).

\subsection{Track Sampling}

The study was conducted at N/a'ankusê Wildlife Sanctuary, located in the Khomas Region of Namibia. The tracks of 8 cheetahs, 4 males and 4 females, were sampled on prepared sandy plots according to the recommendations of Jewell et al. [16]. The cheetahs were all mature adults (adult female (AF) and adult male (AM)) and some were related: AM4, AF3 and AF4, AM1 and AM2, and AF1 and AF2 were from the same litters.

Cheetah tracks were sampled with a Nikon D3200 (24.2 MP) equipped with a Nikkor 18-105 mm f/3.5-5.6 lens (Nikon, Tokyo, Japan). Six photos were taken of each track (Figure 1B), 1 photo from above for the 2D method and 5 photos for the 3D method (1 from above and 4 in the cardinal directions at an angle of $10-15^{\circ}$ from the vertical line). As recommended by Marchal [19], the focal length was kept low (between 30 and $55 \mathrm{~mm}$ ) and constant during each track sampling.

\subsection{Processing of Cheetahs' Tracks}

\subsubsection{D Approach}

Photos for the 2D method were scaled with ImageJ software (https://imagej.nih. gov/, (accessed on 1 March 2021)) by using the centimetric ruler placed next to the track 
(Figure 1B). When needed, the operator decreased the contrast to allow clearer visualization of the track contours.

\subsubsection{D Approach}

All 5 photos were used to reconstruct 3D models of each track using the Agisoft Metashape photogrammetric suite (Agisoft LLC, St. Petersburg, Russia). We used the same reconstruction parameters as in [17] except for the number of key points and tie points (respectively 10,000 and 2000 points) and the reconstruction quality for the dense cloud, which was set on high quality, representing an approximate mean ground sampling distance above $0.0008 \mathrm{~mm}$ per pixel. The 3D models were scaled and optimized using 5 targets automatically located inside the images by the software. These targets were printed on a perpendicular ruler placed next to the track (Figure 1B). Among the 3D models, the tracks were manually segmented from the rest of the model using CloudCompare software (www.cloudcompare.org, (accessed on 1 March 2021)) in order to extract the track from the background. To minimize manipulator biases only one manipulator segmented all 3D models and always observed the following procedure. Firstly, the operator used the bounding box Principal Component Analysis fit function to allow the model to be as perpendicular as possible to the $\mathrm{z}$ axis (representing depth). In order to improve the quality of the segmentation, the model was then colored by depth, highlighting the shape of the track. In the end, the track was manually cut out from the background by using the polygonal selection tool and clicking on the edge of the track to preserve its geometry.

\subsection{Geometric Morphometrics}

\subsubsection{Landmarking}

On each track, 25 landmarks were manually placed on the photos (2D approach) and 3D models according to the guidelines from Jewell et al. (Figure 1D) [16]. The fixed landmark fixed points intended to represent the geometry of the track were displaced at the vertex of the curves of each pads of each track. We used ImageJ for the photos and the Geomorph R package for the 3D models [25]. In addition, surface-sliders (i.e., unfixed landmarks sliding on the model surface) were automatically positioned on the surfaces of each 3D models with the Geomorph package (Figure 1D). In total, we placed 150 surface-sliders.

\subsubsection{Generalized Procrustes Analyses}

Generalized procrustes analyses (GPA) aim to minimize the shape differences between tracks by superimpose the coordinates of the landmark using the least-square distance. It allows removing effects of position, size and rotation between tracks. We performed GPA using the Geomorph package on (i) the entire dataset and (ii) reduced datasets based on foot positions (right hind (RH), left hind (LH), right front (RF), left front (LF)) to reduce the variability induced by different foot positions. This partition allowed us to accentuating the variability caused by sex or identity of the cheetah and therefore enable us to identify these factors more efficiently (more details in Sectopn 2.6, Accuracy Analysis section). GPA allowed us to extract shape (Procrustes coordinates) and size (centroid size) information from the landmark positions.

\subsubsection{Template Consideration}

Geomorph allows the automatic placement of surface-sliders using a template based on one of the studied objects. In order to measure the influence of the chosen template, we tested 2 situations: the use of a single template for all tracks and the use of different templates per foot position. To designate the template, we used the mathematically closest track to the consensus from the GPA with 3D fixed landmark as a template using the findMeanSpec function of the Geomorph $\mathrm{R}$ package. The consensus template allowed us to maximize the differences between tracks in a dataset. 


\subsection{Statistical Analysis}

\subsubsection{Principal Component Analysis}

We ran a principal component analysis (PCA) on the Procrustes coordinates for each recording medium (2D or 3D), landmark configuration (fixed only or fixed with surface-slider), datasets used (entire track set or foot position divided sets) and, for the surface-slider modality the template used (one common template or one template per divided dataset). The results of 19 methodological schemes are described in Figure 2. In the remainder of the paper, we call one set of each modality a combination corresponding to any possible path in Figure 2.

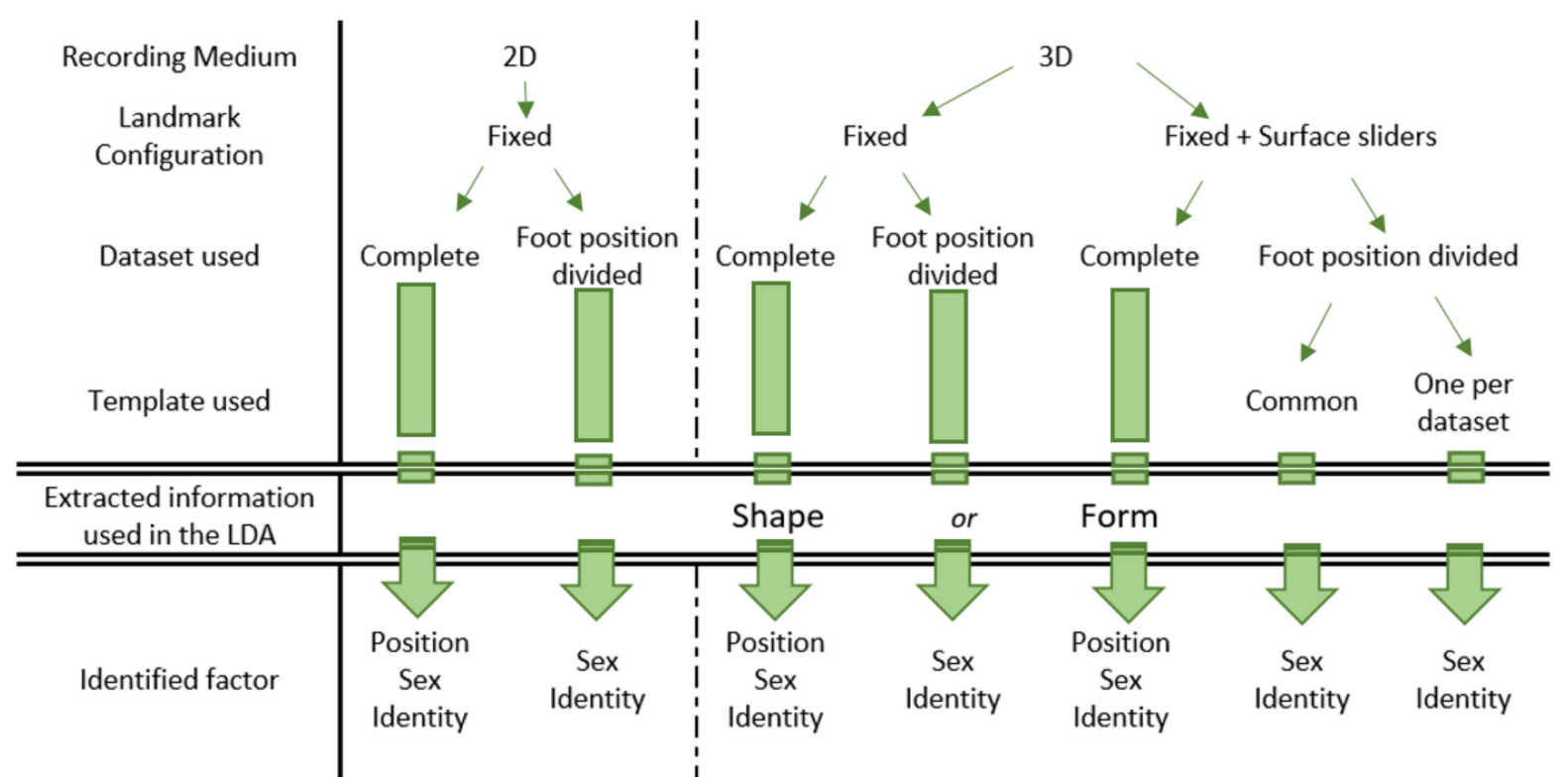

Figure 2. Summary of features implemented in identification algorithm. One set of modalities (recording medium, landmark configuration, dataset and template used) creates a combination. Every possible combination is represented by following green arrows. In all, 19 combinations were obtained and implemented in linear discriminant analysis (LDA), leading to 82 tested identification algorithms (19 combinations $\mathrm{x}$ identification factors $\mathrm{x}$ shape or form information).

\subsubsection{Linear Discriminant Analysis}

Then, we applied a linear discriminant analysis (LDA) with jack-knife prediction by implementing an increasing number of principal components (PCs) to compare the accuracy of prediction for every identification factor (foot position, sex or identity) and every combination tested (Figure 2). We also conducted the same LDA adding centroid size to the PCs in order to use the form information (=size + shape) in the algorithm (Figure 2). That allowed us to compare prediction accuracy using information on the shape only versus information on the form.

\subsection{Accuracy Analysis}

We calculated the total prediction accuracy from the LDA for every possible combination using shape or form information (Figure 2).

Due to the different geometry of the track due to foot position, we believe that an efficient algorithm would need to differentiate the foot position first, and then more precise individual features. This would allow enhancing the contrast related to cheetah's sex or identity within the dataset and therefore enable to identify these factors more efficiently. In order to mimic such an algorithm, we assessed different identification "steps": the first step uses the data from the GPA and its LDA on the entire dataset to predict the foot position of the track (foot position prediction (FPp)). The first identification is combined with the second "step": predicting mean accuracy for sex or identity using the results from LDA 
datasets divided according to foot position (Figure 3). Thus, the total accuracy for these combinations is the foot position identification multiplied by the mean prediction of the divided datasets of the factor (mean factor prediction (MFp)) (Equation (1)). Specifically, for the "one template per foot position" combination, we used the FFp from the "one common template with the entire dataset" combination as the first identification step.

Total accuracy for two step identification $=F P p \times \mathrm{MFp}$.

where

$\mathrm{MFp}=0.25 \times($ LF dataset prediction $+L H$ dataset prediction $+R F$ dataset prediction $+R H$ dataset prediction $)$

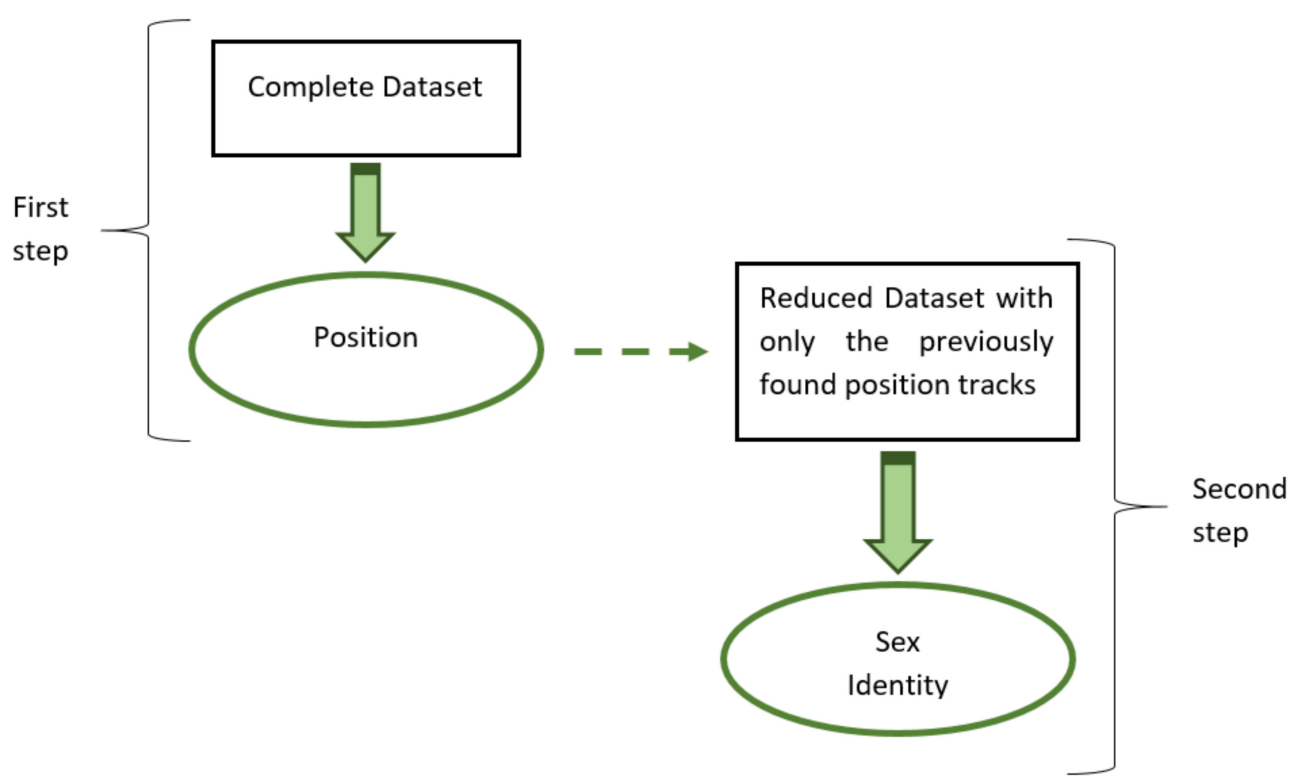

Figure 3. Diagram of "two steps" algorithm. First step: use dataset with entire track collection to assess track position. Second step: use a reduced dataset with track from previously identified position to determine sex or identity.

\section{Results}

We collected 669 tracks corresponding to 18-24 tracks per foot (Table 1). After reconstructing the 3D models and manually segmenting them, the landmarks were positioned on the 3D models. A total of 16,725 fixed landmarks ( 25 per model) and 100,350 surfacesliders (150 per model) were placed on all 3D models $(n=669)$. For the 2D approach, after rescaling, 16,725 landmarks were positioned in total ( 25 per photo). All data extracted from the pictures and 3D models are available by contacting corresponding author or Wildlife 3D Tracking (https://www.wildlife3dtracking.org/, (accessed on 1 March 2021)).

Table 1. Number of sampled tracks per foot position and individual. RF, right front; RH, right hind; LF, left front; LH, left hind; AF, adult female; AM, adult male.

\begin{tabular}{cccccc}
\hline Cheetah & LH & LF & RH & RF & Total \\
\hline AF1 & 22 & 20 & 19 & 20 & 81 \\
AF2 & 18 & 21 & 20 & 24 & 83 \\
AF3 & 20 & 20 & 23 & 24 & 87 \\
AF4 & 23 & 19 & 24 & 21 & 87 \\
AM1 & 20 & 20 & 20 & 20 & 80 \\
AM2 & 20 & 20 & 20 & 20 & 80 \\
AM3 & 21 & 20 & 20 & 20 & 81 \\
AM4 & 23 & 21 & 24 & 22 & 90 \\
\hline Total & 167 & 161 & 170 & 171 & 669 \\
\hline
\end{tabular}




\subsection{Statistical Analysis Results}

For every combination, the first two components of the PCA explained between $43.1 \%$ and $11.8 \%$ and between $15.5 \%$ and $8.6 \%$ of the variation, respectively. The first PC from combinations using the entire dataset accounted for the most variation (between $43.1 \%$ and $35.0 \%$ ) compared to combinations using only the tracks from a specific foot position (between 20.4\% and 11.8\%). Figures 4 and 5 show the PCA (PC1 and PC2) in 3D with form information colorized according to a different factor for each figure. This provides a clear visualization of the differences between points. Figure 4 plots every sampled track and Figure 5 only the right front tracks, to improve visualization of those differences. Figure 4 clearly shows four clusters of points $(n=669)$ corresponding to the four foot positions. Figure 5A is colorized by sex only, while Figure 5B is colorized by individual. We observe that sex is well segregated by PCs (Figure 5A) and specific clusters correspond to individuals or group of individuals (Figure 5B).
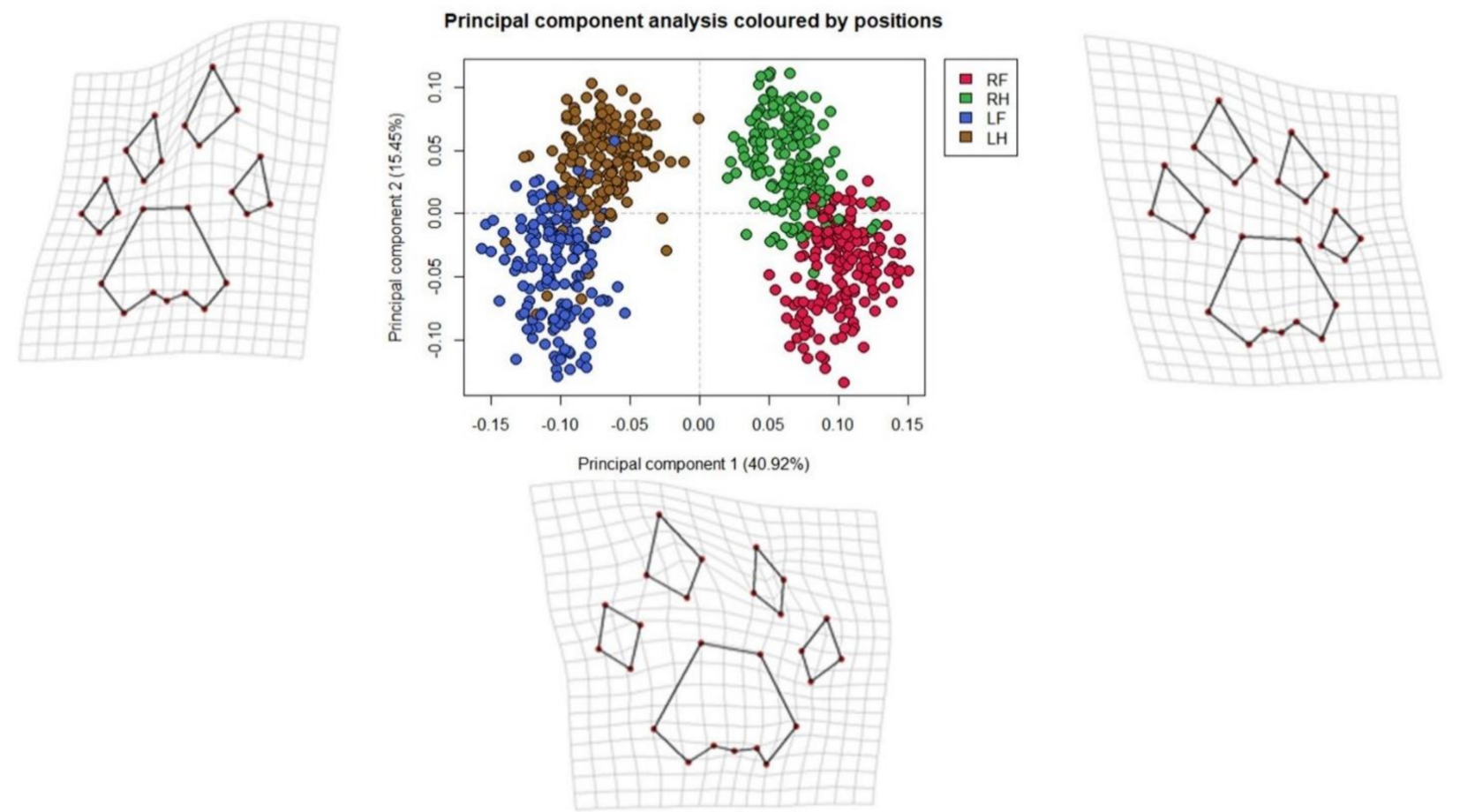

Figure 4. Principal component analysis (PCA) of every sampled track $(n=669)$ with fixed landmark only in 3D and form information colorized by foot position. RF, right front; RH, right hind; LF, left front; LH, left hind. Thin-plate spline deformation grids illustrate differences in shape between tracks corresponding to extremes of each principal component axis and consensus. 
A.
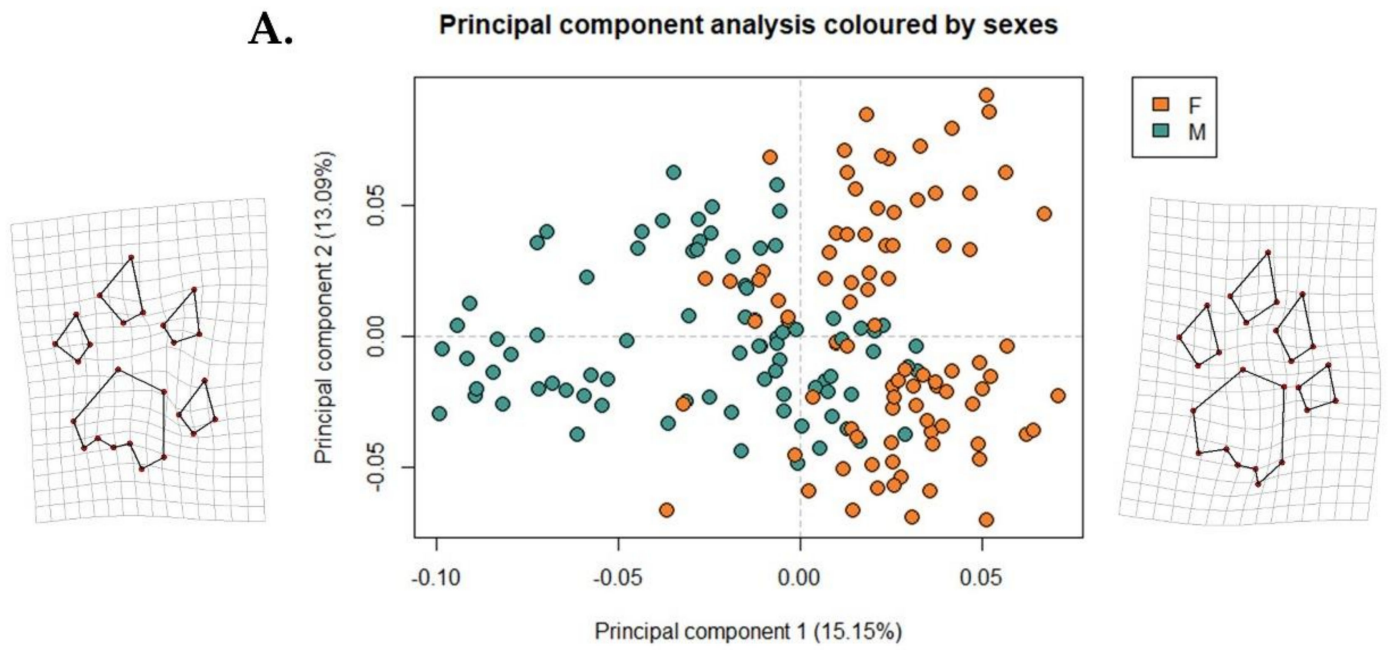

B. Principal component analysis coloured by identities
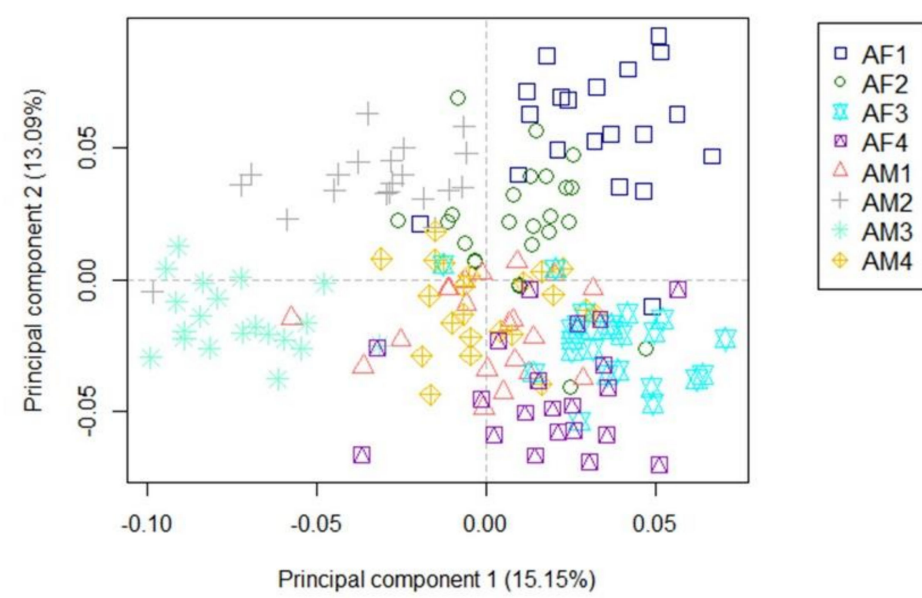

AM4

Figure 5. (A) Principal component analysis of right front (RF) tracks ( $n=171)$ with fixed landmark only in 3D with form information colorized by sex (M, male; $\mathrm{F}$, female). Thin-plate spline deformation grids illustrate differences in shape between tracks corresponding to extremes of first principal component axis and consensus. (B) Principal component analysis of right front $(\mathrm{RF})$ tracks $(\mathrm{n}=171)$ with fixed landmark only in 3D with form information colorized by identity (AM, adult male; AF, adult female).

\subsection{Accuracy Analysis Results}

The maximum prediction accuracy for LDA was assessed for each combination. The accuracy percentage corresponded to the best accuracy obtained with the minimum PCs implemented in LDA. The results show that foot position prediction accuracy was better in 3D than in 2D, with 98.2 and $91.6 \%$ of correct identification, respectively (Table 2). For sex and identity factors, the combinations using all tracks were less accurate than those using datasets divided according to foot position.

The best total accuracy for sex prediction $(94.8 \%)$ is the combination of 3D fixed landmarks with surface-sliders issued from one common template and derived from a form study (Figure 6), while the best total accuracy for identity prediction $(93.7 \%)$ was the same combination using a different template for each foot position. However, the combination using different template modalities was almost as accurate. The differences are 0.1 and $0.4 \%$ for the best sex and identity prediction, respectively. 
Table 2. Maximum prediction accuracy obtained for each combination and factor.

\begin{tabular}{|c|c|c|c|c|c|c|c|c|c|}
\hline \multicolumn{6}{|c|}{ 2D } & \multicolumn{4}{|c|}{$3 \mathrm{D}$} \\
\hline \multicolumn{2}{|c|}{$\begin{array}{c}\text { Landmark } \\
\text { Configuration }\end{array}$} & \multicolumn{2}{|c|}{ Fixed } & \multicolumn{2}{|c|}{ Fixed } & \multicolumn{2}{|c|}{$\begin{array}{c}\text { Fixed }+ \\
\text { Surface-Sliders } \\
\text { (Common Template) }\end{array}$} & \multicolumn{2}{|c|}{$\begin{array}{c}\text { Fixed }+ \\
\text { Surface-Sliders (One } \\
\text { Template per Dataset) }\end{array}$} \\
\hline $\begin{array}{l}\text { Tracks } \\
\text { Used (n) }\end{array}$ & Factor & Shape & Form & Shape & Form & Shape & Form & Shape & Form \\
\hline \multirow{3}{*}{ All (669) } & Position & $91.0 \%$ & $91.6 \%$ & $98.2 \%$ & $98.2 \%$ & $97.9 \%$ & $97.9 \%$ & - & - \\
\hline & Sex & $76.8 \%$ & $87.6 \%$ & $84.5 \%$ & $87.1 \%$ & $86.2 \%$ & $89.4 \%$ & - & - \\
\hline & ID & $57.0 \%$ & $66.5 \%$ & $74.3 \%$ & $75.3 \%$ & $80.7 \%$ & $82.1 \%$ & - & - \\
\hline \multirow{2}{*}{ LF (161) } & Sex & $89.4 \%$ & $91.3 \%$ & $96.9 \%$ & $96.3 \%$ & $98.1 \%$ & $98.1 \%$ & $98.8 \%$ & $98.1 \%$ \\
\hline & ID & $77.6 \%$ & $80.7 \%$ & $90.7 \%$ & $90.7 \%$ & $93.2 \%$ & $93.2 \%$ & $93.8 \%$ & $93.8 \%$ \\
\hline \multirow{2}{*}{ LH (169) } & Sex & $82.6 \%$ & $88.6 \%$ & $88.6 \%$ & $92.8 \%$ & $91.6 \%$ & $95.8 \%$ & $92.2 \%$ & $95.8 \%$ \\
\hline & ID & $80.2 \%$ & $81.4 \%$ & $89.2 \%$ & $92.8 \%$ & $95.2 \%$ & $97.6 \%$ & $97.6 \%$ & $97.6 \%$ \\
\hline \multirow{2}{*}{ RF (171) } & Sex & $95.9 \%$ & $97.7 \%$ & $98.8 \%$ & $98.8 \%$ & $100.0 \%$ & $100.0 \%$ & $100.0 \%$ & $100.0 \%$ \\
\hline & ID & $85.4 \%$ & $88.9 \%$ & $94.2 \%$ & $93.6 \%$ & $94.7 \%$ & $94.2 \%$ & $95.3 \%$ & $95.3 \%$ \\
\hline \multirow{2}{*}{ RH (170) } & Sex & $80.0 \%$ & $90.0 \%$ & $90.0 \%$ & $89.4 \%$ & $92.9 \%$ & $93.5 \%$ & $92.9 \%$ & $92.9 \%$ \\
\hline & ID & $84.7 \%$ & $90.0 \%$ & $94.1 \%$ & $94.7 \%$ & $96.5 \%$ & $96.5 \%$ & $96.5 \%$ & $96.5 \%$ \\
\hline
\end{tabular}

A

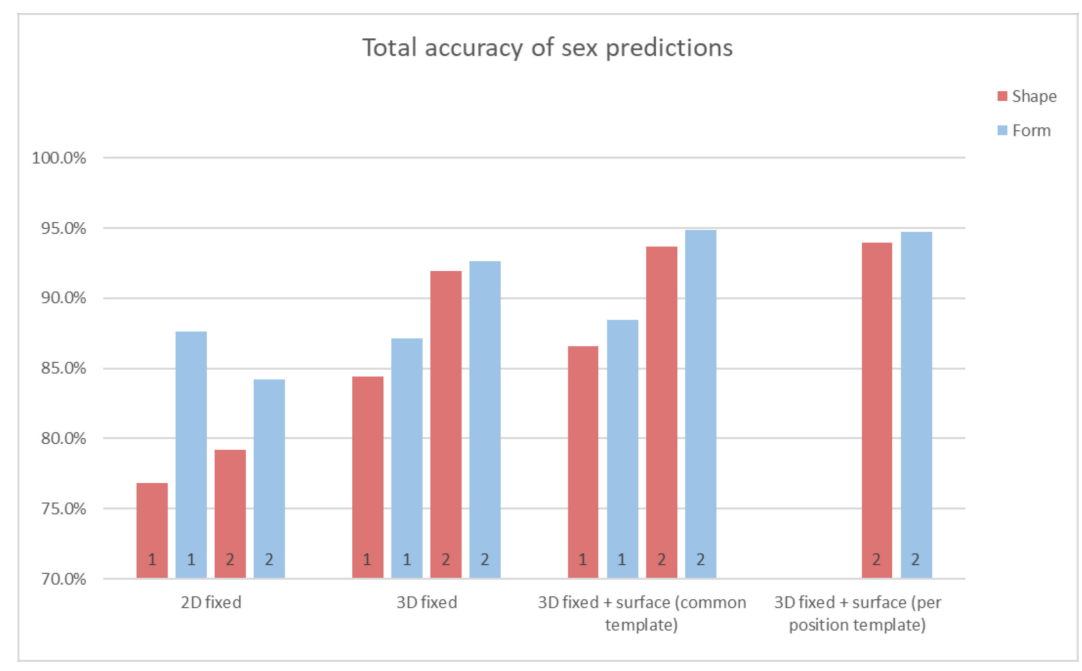

B

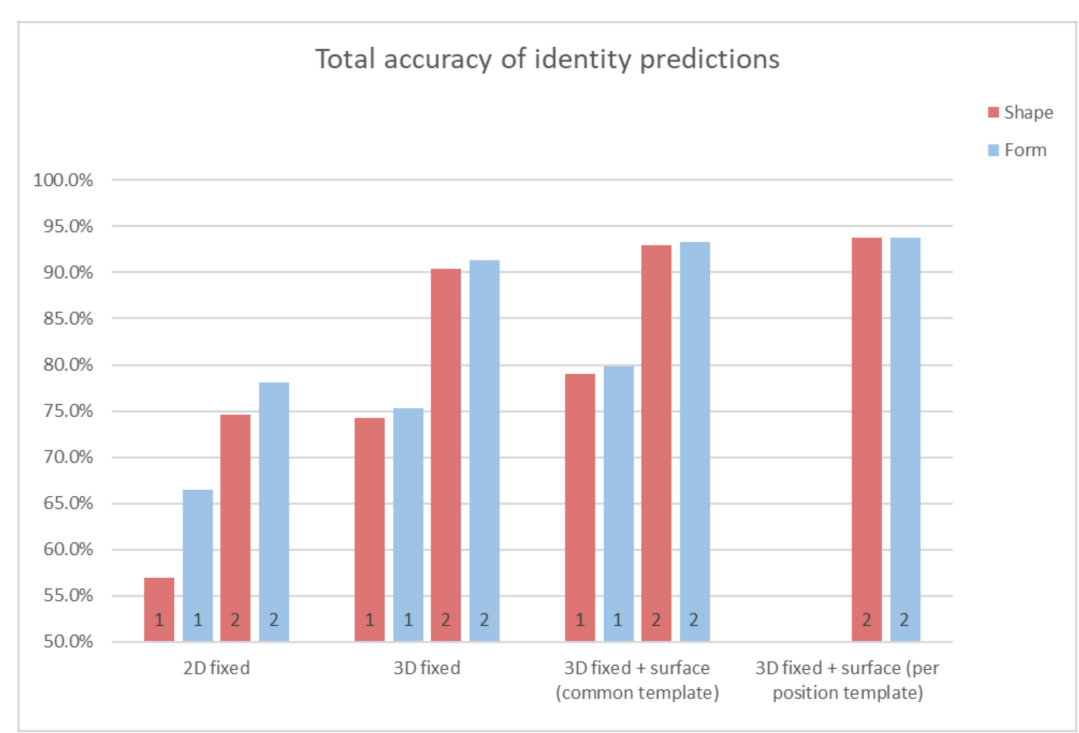

Figure 6. (A) Total accuracy for sex prediction. (B) Total accuracy for identity prediction. Numbers inside bars correspond to number of identification "steps" in the combination: $1=$ all tracks used directly for prediction, 2 = foot position prediction for entire track set combined to identity prediction with datasets already divided by foot position (i.e., two steps: identify foot position and identify identity in dataset of previously found foot position). 
In addition, overall prediction accuracy was better with two identification steps for sex and identity factors. LDA results using either shape or form variables were similar. The combination using form had a slightly better prediction rate: mean difference was 3.3 for sex and $2.3 \%$ for identity factors.

\section{Discussion}

During this experiment, we achieved the identification of individual features of adult cheetahs using different combinations of modalities (Figure 2). Firstly, differentiation of each foot was successfully accomplished. For 3D, about $98 \%$ of positions were correctly discriminated by LDA over the entire dataset. Such a high rate makes it possible to consider using a second identification step based on datasets divided by the previously found foot positions. Predictions on sex and identity remained imprecise for combinations using the entire track set (Figure 6). This is certainly due to the difference in morphology between tracks.

Two factors are involved: the foot position and the size of the pads, which depends on right-left symmetry and the length of the paw impression on the substrate. Cheetahs' front paws are wider, and their hind paws are larger and longer. We can observe that PC1 and PC2 separate right and left more efficiently than front and hind positions (Figure 4). This is also probably due to the impression quality of the track on the ground. When the substrate is too soft, we noticed that the tracks appeared to be longer because of the weight transfer on the leg when the animal is moving, making front tracks look like hind track. Furthermore, sexual dimorphism also generates statistical noise, as males are larger than females (Figure 5A, Marker et al. [26]) and the front feet of a male can be mistaken for the hind feet of a female if the track impression is not optimal. The distinction between front and hind tracks for females is also more visually subtle because the hind track elongation is less pronounced.

Here, the quality of the used tracks was optimal due to the sampling conditions. In a natural environment, the tracks are prone to degrade quickly under the influence of many factors, such as humidity, substrate, the animal's gait or weather conditions [10]. During this study, the tracks were sampled in moistened and smoothed sand patches. Furthermore, sand aggregates falling inside the track were gently blown away. As this tool is intended to be used under real conditions, we recommend that future studies test different substrates and gaits to verify their impact on predictions. Besides the prediction accuracy, these factors can induce variability in the segmentation of tracks and thus on the positioning of landmarks. Loose impressions in the substrate or deformed tracks due to cheetah movement present poorly defined contours, especially for lower part of the main pad. The segmentation, even colored by depth, is then subjected to operator bias. In our case, we reduced the possible influence of this bias by working with a single operator.

To overcome the variability induced by the foot positions discussed above, we separated the dataset into four subsets depending on foot positions. The accuracy increased, because these datasets highlighted the variability between tracks from the same foot of different individuals, thus the variability related to sex and identity. A sex sub-division of these datasets was performed, but the identity identification results decreased. This may be due to the small number of cheetahs remaining in these datasets. Furthermore, we can observe that the best results were obtained with fixed landmarks coupled with surface-sliders (Figure 6). The influence of the number of templates used is relatively limited and could indicate that the number of surface-sliders is too high. We believe that with more individuals implemented in the dataset, the differences would be more significant. Curve-sliders could also be placed on the edges of the tracks. Nevertheless, the edges are dependent of the depth of the track, making them relatively variable between tracks. In the same way, as 3D models are polygonal meshes, the manual removal of tracks can induce additional noise from the irregularities of the remaining mesh faces. We believe that using curve-sliders would have led to more statistical noise than usable information and made the method more tedious. 
The graph of PCs 1 and 2 for the front right tracks (Figure 5B) shows that some individuals are more clearly segregated from others. This separation seems to correspond to the general size of the individuals. For example, AM3 is the largest cheetah in this dataset. His tracks appear as a cluster distinct from the others. It would be interesting to test the relationship between the form of the track and the body condition of the animal in further studies. Links between track form and morphological features such as body mass are being investigated for different species and for estimation of features of extinct animals [27]. This algorithm might allow a more precise estimation of the morphological parameters of cheetahs such as their overall size or weight. Such information would be valuable for scientists in charge of setting up conservation strategies specifically for elusive carnivores [14,15]. Besides, AM4 is a smaller male, and PC1 and PC2 hardly distinguish it from females.

In addition, it should be noted that for two of the three groups of cheetahs (AF1-AF2 and AF3-AF4-AM4) from the same litter, the scores of related individuals are juxtaposed or superimposed. As well, the cluster formed by AM3, not related to any other cheetah, is well segregated from the others. To some extent, it is conceivable that the morphology of the track is a hereditary trait and might be evidence of the animal's genetics. Thus, this method could potentially assess the relatedness between cheetahs.

Differences among results using information from shape or form remain relatively small (Table 2, Figure 6). Therefore, size information seems to be of limited relevance for the morphometric study of adult cheetah tracks. Further analysis of size should include more individual cheetahs to assess the true impact of size on predictions, especially with different age groups. Size may have a significant influence when discriminating animal age. Methods implying size are already being used to estimate age and population age structure but sometimes remain imprecise $[19,28]$. Concerning the variation in the geometry of the tracks according to age, the track being dependent on the anatomy of the leg and foot, as well as the gait, the ontogeny of the animal can have an effect on its shape. The gait changes for the domestic cat (Felis catus) as the animal's neuromuscular development change from plantigrade or semi-plantigrade to digitigrade as an adult [29]. A similar development in cheetahs could influence the tracks left by animals of different age groups. This question could be answered by following different animals throughout their lives and comparing their tracks. However, geometric morphometry is a technique that eliminates the effects of size and only keeps the differences in pure geometry. Using only shape data, the differences according to age could be minimized, therefore enabling individual identification throughout the entire life of individuals. In addition, the use of the shape alone would make it possible to ignore the use of a scale, since GPA eliminates any effect of scale, position and orientation. Thus it simplifies fieldwork by removing the necessity of having a ruler. The user would then need only a camera, which is readily available thanks to the existence of smartphones.

Using photography and 3D models to differentiate and identify species or individuals is the main goal of both FIT and the method explained in this paper. FIT has already proven its efficacy in discriminating individual features in various mammals [15]. However, some limitations might exist when it comes to tracks with limited geometrical variety. Indeed, morphometrics studies permit us to extract information related to the form of the track. As carnivores and rodents have complex tracks with digits and pads, the quantity of information extracted is considerably larger and the identification is more likely to be successful $[16,30,31]$. In another way, other animal such as ungulate mammals tend to have simpler tracks from a geometrical point of view, and identification would then seem to be more challenging regarding the lack of mathematical information [10]. However, it would be possible to determine morphological features such as age and sex thanks to size variations and sexual dimorphism. Here, we believe that 3D would be of great interest: depth information of the 3D model could be used to estimate features such as the body mass. In order to achieve that, the relationship between the tracks and body features of studied species is discussed in various studies $[27,28,30]$. In this study, we did not have 
access to the weight of the animals. However, cheetahs are animals with a clear sexual dimorphism, the males being larger and heavier than the females [26]. The efficiency of sex identification allows us to consider the possibility of estimating the weight of an individual based on its tracks. On the other hand, it is also possible that a significant variation in the weight of an individual can induce differences in the track and lead to misidentifications, as cheetahs are animals that can undergo significant weight variations due to their environment. In addition, substrate conditions such as composition or wetness can have an impact on the quality of the imprint and thus the geometry of the modelled tracks. Similarly, the tracks will be different depending on the gait of the animal. Tracks left during a run are unusable because of their poor quality. To overcome these problems, we used controlled conditions (i.e., prepared sand patch) to limit as much as possible the differences linked to the substrate. The cheetahs were always at a slow pace, following the operator in possession of the meat reward. At the end of the sampling, only good quality prints were kept. To overcome limitations related to track quality in the field, we recommend collecting cheetah tracks near riverbeds or on sandy roads, with little or no stones, or after rainy episodes. Wet soils allow for a better impression of the foot in the substrate. In addition, animals are more likely to walk slowly near water, for example to come and drink. The probability of finding good quality tracks therefore tends to be higher. Nevertheless, tracks variation depending on substrate conditions and animal's gait still needs to be investigated.

The best identification algorithm allows $93.7 \%$ of individuals to be distinguished (Figure 6B). By way of comparison, the FIT approach with cheetahs also has a success rate of more than $90 \%$ [16]. However, FIT only uses the left hind tracks. The tool under development in this study can identify individuals based on any cheetah track. The FIT algorithm is also calibrated with more data (38 cheetahs sampled). A trial with as many individuals would be necessary to verify which technique is the most efficient. As LDA is a supervised classification method, a comparison with an unsupervised classification method would highlight the efficiency of geometric morphometrics studies to discriminate individual characteristics extracted from tracks. In addition, Table 2 shows that front tracks facilitate the separation of sex, while hind tracks facilitate the separation of individuals (mean for sex predictions is $97.4 \%$ for front and $90.6 \%$ for hind tracks; mean for identity predictions is $90.8 \%$ for front and $92.6 \%$ for hind tracks). These observations are related to the sexual dimorphism of cheetah feet, as explained above. On the one hand, males have more massive paws than females. The variability explained by the PC incorporated in the LDA explains this difference. On the other hand, hind tracks are more similar between males and females. The variability thus emphasizes the individual differences.

Another major difference from FIT is the use of 3D models versus purely 2D photos. The FIT technique is therefore comparatively easier to apply. Nevertheless, the differences between our 2D and 3D algorithms show better predictions with 3D data. The principal limitations of the 3D approaches lie in the processing time for model reconstruction and manual segmentation. These steps are time-consuming, especially for large datasets. However, the possibility of using cloud computing would overcome the first limitation by providing access to computers powerful enough to make 3D reconstruction virtually instantaneous. The recent development of LiDAR sensors integrated in smartphones is also very promising as they allow instantaneous 3D perception. For segmentation, an approach using machine learning should be able to separate the tracks from the substrate. It would need to first recognize tracks based on their geometry and depth information, and segment them from the model. This technique is being developed for 2.5D by FIT [32] with limited success. We believe that $3 \mathrm{D}$ would obtain better results. The positioning of landmarks could also be automated by using the geometry of the track. The placement of landmarks (type II) corresponds to the extrema curves of local structures that could be identified by trained algorithms [23]. Ultimately, track recognition software such as a citizen-science smartphone application could be developed using only five track photos as input. The pictures could be geo-referenced thanks to the integrated GPS of the cellphone. 
The software would place the landmarks depending on the geometry of the track parts and allow for the identification of animals from different species, along with their sex and identity. It would provide accurate and valuable information to conservation scientists, inform tourists or local community members, and raise awareness about the status of identified animals.

\section{Conclusions}

To conclude, the use of close-range remote sensing and morphometry turns out to be effective at extracting mathematical data from tracks, which can then be translated into individual traits. With a greater volume of data and by automating the process, this tool could enable managers and conservationists of protected areas to obtain precise information on the population status of elusive animals that are difficult to inventory in the conventional way, such as carnivores. This non-invasive approach decreases the intrusion on the study animals and is cost-effective. The method can potentially be extended to any species, including humans (for anti-poaching efforts), that present idiosyncratic foot morphology. It would, therefore, have an impact on various fields such as conservation biology, wildlife management, human-wildlife conflict and anti-poaching.

Author Contributions: A.F.J.M., A.M. and G.B. designed the methodology, G.B. collected the data, G.B. analyzed the data and discussed the results. G.B. and A.M. wrote the manuscript according to the feedbacks given by all authors. All authors have read and agreed to the published version of the manuscript.

Funding: This research was funded by The Rufford Foundation, grant number 24248-2.

Data Availability Statement: Not applicable.

Acknowledgments: We thank the N/a'ankusê Foundation for letting us conduct this study with their semi-captive cheetah and for their expertise in terms of track sampling. Special thanks to Hanlo Fouche, Kobus and Lauren Hoffman, Stuart Munro, Jule Piloth and Megan Sparrow for the sampling mornings.

Conflicts of Interest: The authors declare no conflict of interest.

\section{References}

1. Vitousek, P.; Mooney, H.A.; Lubchenco, J.; Mellilo, J.M. Human Domination of Earth. Science 1997, 227, 494-499. [CrossRef]

2. Ceballos, G.; Ehrlich, P.R.; Dirzo, R. Biological Annihilation via the Ongoing Sixth Mass Extinction Signaled by Vertebrate Population Losses and Declines. Proc. Natl. Acad. Sci. USA 2017, 114, E6089-E6096. [CrossRef] [PubMed]

3. Cardillo, M.; Purvis, A.; Sechrest, W.; Gittleman, J.L.; Bielby, J.; Mace, G.M. Human Population Density and Extinction Risk in the World's Carnivores. PLoS Biol. 2004, 2, e197. [CrossRef]

4. Karanth, K.U.; Chellam, R. Carnivore Conservation at the Crossroads. ORYX 2009, 43, 1-2. [CrossRef]

5. Durant, S.; Mitchell, N.; Ipavec, A.; Groom, R. Acinonyx Jubatus (Cheetah). IUCN Red List 2015. [CrossRef]

6. Durant, S.M.; Mitchell, N.; Groom, R.; Pettorelli, N.; Ipavec, A.; Jacobson, A.P.; Woodroffe, R.; Böhm, M.; Hunter, L.T.B.; Becker, M.S.; et al. The Global Decline of Cheetah Acinonyx Jubatus and What It Means for Conservation. Proc. Natl. Acad. Sci. USA 2017, 114, 528-533. [CrossRef]

7. Marker, L.; Cristescu, B.; Dickman, A.; Nghikembua, M.T.; Boast, L.K.; Morrison, T.; Melzheimer, J.; Fabiano, E.; Mills, G.; Wachter, B.; et al. Ecology of Free-Ranging Cheetahs. In Cheetahs: Biology and Conservation; Biodiversity of the World: Conservation from Genes to Landscapes; Academic Press: Cambridge, MA, USA, 2017; pp. 107-119, ISBN 9780128041208.

8. West, G.; Heard, D.; Caulkett, N. Zoo Animal and Wildlife Immobilization and Anesthesia, 2nd ed.; John Wiley and Sons: Hoboken, NJ, USA, 2014; ISBN 9781118792919.

9. Kauffman, M.J.; Sanjayan, M.; Lowenstein, J.; Nelson, A.; Jeo, R.M.; Crooks, K.R. Remote Camera-Trap Methods and Analyses Reveal Impacts of Rangeland Management on Namibian Carnivore Communities. Oryx 2007, 41, 70. [CrossRef]

10. Liebenberg, L. A Field Guide to Animal Tracks of Southern Africa; David Philip Publishers: Cape Town, South Africa; Johannesburg, South Africa, 1990.

11. Liebenberg, L. The Art of Tracking, the Origin of Science; David Philip Publishers: Cape Town, South Africa, 1990.

12. Stander, P.E.; Ghau, I.I.; Tsisaba, D.; Oma, I.I., VI. Tracking and the Interpretation of Spoor: A Scientifically Sound Method in Ecology. J. Zool. 1997, 242, 329-341. [CrossRef]

13. Smallwood, K.S.; Fitzhugh, E.L. A Rigorous Technique for Identifying Individual Mountain Lions Felis Concolor by Their Tracks. Biol. Conserv. 1993, 65, 51-59. [CrossRef] 
14. Alibhai, S.K.; Jewell, Z.C.; Law, P.R. A Footprint Technique to Identify White Rhino Ceratotherium Simum at Individual and Species Levels. Endanger. Species Res. 2008, 4, 205-218. [CrossRef]

15. Alibhai, S.; Jewell, Z.; Evans, J. The Challenge of Monitoring Elusive Large Carnivores: An Accurate and Cost-Effective Tool to Identify and Sex Pumas (Puma Concolor) from Footprints. PLoS ONE 2017, 12, e0172065. [CrossRef] [PubMed]

16. Jewell, Z.; Alibhai, S. Identifying Endangered Species from Footprints. SPIE 2012. [CrossRef]

17. Jewell, Z.C.; Alibhai, S.K.; Weise, F.; Munro, S.; Van Vuuren, M.; Van Vuuren, R. Spotting Cheetahs: Identifying Individuals by Their Footprints. J. Vis. Exp. 2016, 2016. [CrossRef]

18. Marchal, A.F.J.; Lejeune, P.; de Bruyn, P.J.N. Virtual Plaster Cast: Digital 3D Modelling of Lion Paws and Tracks Using Close-Range Photogrammetry. J. Zool. 2016, 300, 111-119. [CrossRef]

19. Marchal, A.F.J.; Lejeune, P.; Bruyn, P.J.N. de Identification of the Anteroposterior and Mediolateral Position of Lion Paws and Tracks Using 3D Geometric Morphometrics. Afr. J. Wildl. Res. 2017, 47, 106-113. [CrossRef]

20. Marchal, A.F.J. Monitoring Lions (Panthera Leo) Using Digital 3D Models of Their Tracks. Ph.D. Thesis, Université de Liège, Liège, Belgium, 2017.

21. Zelditch, M.L.; Swiderski, D.L.; Sheets, H.D.; Fink, W.L. Geometric Morphometrics for Biologists: A Primer; Academic Press: London, UK; Waltham, MA, USA; San Diego, CA, USA, 2004; Volume 95, ISBN 9780127784601.

22. Mitteroecker, P.; Gunz, P. Advances in Geometric Morphometrics. Evol. Biol. 2009, 36, 235-247. [CrossRef]

23. Gunz, P.; Mitteroecker, P. Semilandmarks: A Method for Quantifying Curves and Surfaces. Hystrix 2013, 24. [CrossRef]

24. Bookstein, F.L. Morphometric Tools for Landmark Data: Geometry and Biology; Cambridge University Press: Cambridge, UK, 1991.

25. Cucchi, T.; Baylac, M.; Evin, A.; Bignon-Lau, O.; Vigne, J.-D. Morphométrie géométrique et archéozoologie: Concepts, méthodes et applications. In Messages d'os Archéométrie du Squelette; Editions des Archives Contemporaines: Paris, France, 2015; ISBN 9782813001641.

26. Sherratt, E. Quick Guide to Geomorph v.2.1.6. 2015, pp. 1-99. Available online: https://www.researchgate.net/profile/Ariel_ Marcy/post/Morphometry_and_convergence_divergence/attachment/59d63b0879197b8077998163/AS\%3A408679657558016 \%401474448231372/download/Quick_Guide_to_Geomorph_v2.1.pdf (accessed on 1 March 2021).

27. Marker, L.L.; Dickman, A.J. Morphology, Physical Condition, and Growth of the Cheetah (Acinonyx Jubatus Jubatus). J. Mammal. 2003, 84, 840-850. [CrossRef]

28. Schanz, T.; Lins, Y.; Viefhaus, H.; Barciaga, T.; Läbe, S.; Preuschoft, H.; Witzel, U.; Sander, P.M.; Dodson, P. Quantitative Interpretation of Tracks for Determination of Body Mass. PLoS ONE 2013, 8, e77606. [CrossRef]

29. Western, D.; Moss, C.; Georgiadis, N. Age Estimation and Population Age Structure of Elephants from Footprint Dimensions. J. Wildl. Manag. 1983, 47, 1192. [CrossRef]

30. Peters, S.E. Postnatal Development of Gait Behaviour and Functional Allometry in the Domestic Cat (Felis Catus). J. Zool. 1983, 199, 461-486. [CrossRef]

31. Gu, J.; Alibhai, S.K.; Jewell, Z.C.; Jiang, G.; Ma, J. Sex Determination of Amur Tigers (Panthera Tigris Altaica) from Footprints in Snow. Wildl. Soc. Bull. 2014, 38, 495-502. [CrossRef]

32. Russell, J.C.; Hasler, N.; Klette, R.; Rosenhahn, B. Automatic Track Recognition of Footprints for Identifying Cryptic Species. Ecology 2009, 90, 2007-2013. [CrossRef] [PubMed] 